\title{
Temperature dependence of the cosphi conductance in Josephson tunnel junctions determined from plasma resonance experiments
}

\author{
Pedersen, Niels Falsig; Sørensen, O. H.; Mygind, Jesper
}

Published in:

Physical Review B

Link to article, DOI:

10.1103/PhysRevB.18.3220

Publication date:

1978

Document Version

Publisher's PDF, also known as Version of record

Link back to DTU Orbit

Citation (APA):

Pedersen, N. F., Sørensen, O. H., \& Mygind, J. (1978). Temperature dependence of the cosphi conductance in Josephson tunnel junctions determined from plasma resonance experiments. Physical Review B, 18(7), 32203230. https://doi.org/10.1103/PhysRevB.18.3220

\section{General rights}

Copyright and moral rights for the publications made accessible in the public portal are retained by the authors and/or other copyright owners and it is a condition of accessing publications that users recognise and abide by the legal requirements associated with these rights.

- Users may download and print one copy of any publication from the public portal for the purpose of private study or research.

- You may not further distribute the material or use it for any profit-making activity or commercial gain

- You may freely distribute the URL identifying the publication in the public portal 


\title{
Temperature dependence of the $\cos \phi$ conductance in Josephson tunnel junctions determined from plasma resonance experiments
}

\author{
N. F. Pedersen, O. H. Soerensen, and J. Mygind \\ Physics Laboratory I, The Technical University of Denmark, DK-2800 Lyngby, Denmark
}

(Received 31 October 1977)

\begin{abstract}
The microwave response at $9 \mathrm{GHz}$ of Sn-O-Sn tunnel-junction current biasęd at zero dc voltage has been measured just below the critical temperature $T_{c}$ of the Sn films. The temperature dependence of the $\cos \phi$ conductance is determined from the resonant response at the junction plasma frequency $f_{p}$ as the temperature is decreased from $T_{c}$. We used three different schemes for observation of the plasma oscillations: (a) secondharmonic generation (excitation at $\sim 4.5 \mathrm{GHz}, f_{p} \sim 4.5 \mathrm{GHz}$ ); (b) mixing (excitations at $\sim 9$ and $\sim 18 \mathrm{GHz}$, $f_{p} \sim 9 \mathrm{GHz}$ ); (c) parametric half-harmonic oscillation (excitation at $\sim 18 \mathrm{GHz}, f_{p} \sim 9 \mathrm{GHz}$ ). Measurements were possible in two temperature intervals, $0.994 \leq T / T_{c} \leq 0.982$ and $0.965 \geq T / T_{c} \geq 0.930$, with the result that as the temperature was decreased, the $\cos \phi$ amplitude first increased from about zero to positive values and then at lower temperatures decreased approaching -1 at the lowest temperatures of the experiment.
\end{abstract}

\section{INTRODUCTION}

The existence of a phase-dependent Ohmic current in Josephson tunnel junctions was experimentally verified by Pedersen et al. ${ }^{1}$ They found that "the quasiparticle-pair interference tunnel current predicted by Josephson exists and has the expected magnitude but the opposite sign." According to the tunneling theory of Josephson, ${ }^{2}$ which later has been"worked out in detail by Poulsen ${ }^{3}$ and Harris, ${ }^{4}$ the sign of the phase-dependent conductance is positive, i.e., in phase with the quasiparticle contribution. More recently, the phasedependent conductance also known as the $\cos \phi$ conductance or the $\cos \phi$ term has been observed in virtually every type of Josephson junction: Point contacts, ${ }^{5}$ Dayem bridges,${ }^{6}$ Solder-blob junctions, ${ }^{7}$ and also tunnel junctions. ${ }^{8}$ In all cases the experiments were interpreted as evidence for the existence of a $\cos \phi$ conductance with a negative sign, in agreement with the early result of Pedersen et al. ${ }^{1}$

This striking discrepancy between the tunneling theory and the experiments has remained unresolved. Alternative theories which take into account the finite relaxation time of the superconducting order parameter ${ }^{9}$ most often based on time-dependent Ginzburg-Landau theory ${ }^{10,11}$ have lead to a negative $\cos \phi$ term in agreement with the experiments. In view of these results it seems reasonable to conclude that the phase-dependent conductance predicted by tunneling Hamiltonian calculations has in fact not yet been observed and that relaxation effects must be included in order to explain the experimental results.

In an attempt to provide additional information on the origin of the $\cos \phi$ conductance we have made measurements on tunnel junctions as a func- tion of temperature very near the superconducting transition temperature $T_{c}$.

We have studied the resonant microwave response of the current biased junctions at zero dc voltage. A resonant response may be observed when a microwave signal containing one (two) frequency component $(s)$ is applied to the junction and the junction plasma frequency $f_{p}$, by means of the dc bias current is tuned to coincide with one of the applied frequencies, with harmonics or subharmonics of these or with their sum and difference frequencies. In this way we have measured the response at $\sim 9 \mathrm{GHz}$ using (a) the second harmonic of an excitation at $\sim 4.5 \mathrm{GHz}$, (b) the difference frequency component with excitations at $\sim 9$ and $\sim 18 \mathrm{GHz}$ applied simultaneously, and (c) the half harmonic parametric oscillation with an excitation at $\sim 18 \mathrm{GHz}$. In all three cases the excitation(s) and the junction response were separated in frequency.

The paper is organized as follows: In Sec. II the experimental methods are described in detail. Section III contains an account of the theoretical evaluation leading to expressions for the measured quantities which are compared to the experimental results in Sec. IV. Finally, Sec. V presents the conclusion and a brief summary of the paper.

\section{EXPERIMENTAL METHODS}

The junctions used in the experiment were standard Sn-O-Sn tunnel junctions evaporated onto 1mm-thick microscope slides. The overlap areas were typically $0.2 \times 0.1 \mathrm{~mm}$. The SnO barrier was grown on top of the Sn film by thermal oxidation for about 16 hours which resulted in junction resistances of order $0.1-0.3 \Omega$. The junctions had current densities of about $20 \mathrm{~A} / \mathrm{cm}^{2}$ and $R I_{c}$ pro- 


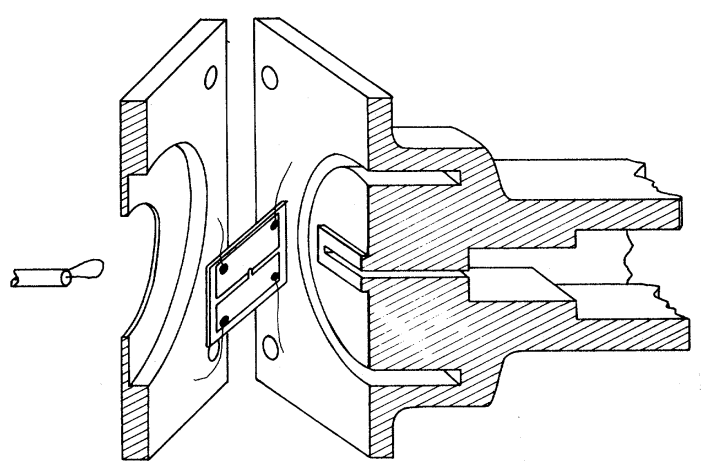

FIG. 1. Cut away drawing of the impedance transformer and the junction mounting. The mylar foil providing the dc insulation between junction and waveguide is not shown.

ducts within $90 \%$ of the theoretical maximum ( $\pi \Delta /$ $2 e$ ) when extrapolated to $T=0 \mathrm{~K}$. Here, $R$ is the normal-state junction resistance, $I_{c}$ the critical current, and $2 \Delta / e$ the energy gap in eV. Selfinduced steps due to internal geometrical resonances ${ }^{12}$ were not observable in the $I-V$ curves of our junctions at voltages less than $\sim 50 \mu \mathrm{V}$.

The junction geometry and the mounting across the end of a two section binomial quarter-wave microwave transformer is shown in Fig. 1. The height of the low impedance end is $0.4 \mathrm{~mm}$ and a $25 \mu \mathrm{m}$ mylar foil is inserted to provide dc insulation between the junction and the flange of the transformer. By applying a microwave signal to the junction through the transformer we have found from the height of the microwave induced steps that of order $10 \%$ of the incident power entered the junction. This efficient coupling was only achieved within a $100-\mathrm{MHz}$ frequency band, much smaller than the inherent bandwidth of the transformer. Less than $5 \%$ of the incident power was reflected in the same narrow bandwidth.

The junction was directly immersed in liquid helium. The temperature in the cryostat was regulated by pumping on the helium surface through a standard manostat. By using a stabilized reference pressure the rate of change of the helium gas pressure was below 0.1 Torr per hour. This stability was sufficient to ensure that no observable change of the maximum supercurrent occurred during an experimental run.

The maximum supercurrent or critical current both with and without applied microwaves was carefully measured. Since all experiments reported here were performed just below the transition temperature the critical current was rather sensitive to fluctuations. In order to determine the critical current we traced out $\sim 100$ consecutive $I-V$ curves on a storage oscilloscope at a rate of
5-10 traces/sec. Due to fluctuation effects we usually observed a $5 \%$ spread of the maximum supercurrent value and we identified the largest recorded value with the critical current. The critical current measured in this way could be reproduced within $0.1 \%$. The relative microwave induced suppression of the critical current was an important input parameter in the calculation of the nonlinear junction response as discussed in Sec. III.

Microwave signals at $\sim 4.5, \sim 9$, and $\sim 18 \mathrm{GHz}$ were applied to the junction via a semirigid coaxial line terminated in a loop placed in a nonresonant structure behind the junction substrate (as indicated in Fig. 1). Only a small fraction of the power applied to the loop reached the junction. The microwave sources were all frequency locked

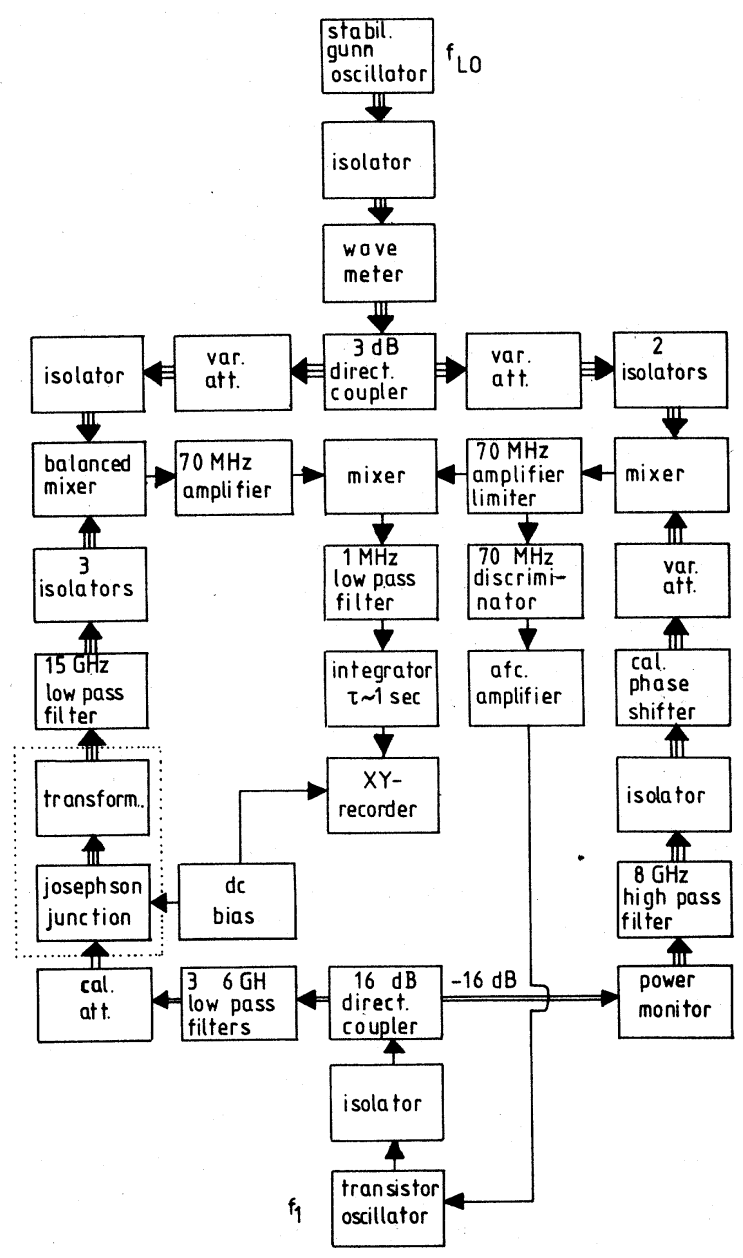

FIG. 2. Diagram of the microwave system for secondharmonic generation. $\left[f_{1}=\frac{1}{2}\left(f_{\text {LO }} \pm f_{\text {i.f. }}\right)\right]$. Triple line: wave guide. Double line: microwave coaxial cable. Single line: low-frequency coaxial cable. AFC: automatic frequency control. 
to the local oscillator ( $\mathrm{LO})$ of the receiver. The frequency of the LO (an $X$-band Gunn-diode oscillator) was stabilized to better than $2 \times 10^{-6}$ and showed a long-term drift less than $1 \mathrm{MHz}$ per day. No dc magnetic field was applied during the experiments and two $\mu$-metal cans reduced the ambient magnetic field to a few millioersted. The measurements were performed in a shielded room and in addition the cryostat was enclosed in a copper can and equiped with electrical filters on all leads.

\section{A. Second harmonic generation}

The microwave interferometer used for detec-: tion of the second harmonic voltage generated by the junction is shown schematically in Fig. 2. The excitation signal at $f_{1} \sim 4.5 \mathrm{GHz}$ derived from a 3-5 GHz transistor oscillator was fed to the junction through an isolator, three low-pass filters (cutoff at $6 \mathrm{GHz}$, total attenuation in excess of $180 \mathrm{~dB}$ at $8 \mathrm{GHz}$ ), a variable attenuator and the coupling loop. The second harmonic signal generated by the junction was detected in the balanced mixer producing an intermediate frequency (i.f.) of $70 \mathrm{MHz}$. A reference signal also at $70 \mathrm{MHz}$ was obtained by mixing the LO with the second harmonic of the transistor oscillator. Finally, the two coherent i.f. signals were mixed and the dc output was filtered and led to the $Y$-axis of an $X-Y$ recorder. The $X$ axis was controlled by the junction dc-bias current. By adjusting the $X$-band phase shifter in the reference arm any projection of the second harmonic voltage may be recorded. Selecting, for instance, two mutually orthogonal settings of the phase shifter both amplitude and phase of the second harmonic response may be determined. The sensitivity of this detection scheme was $\sim 4 \times 10^{-20}$ $W$ in a $1-\mathrm{Hz}$ bandwidth.

\section{B. Mixing}

The tunnel junction biased at zero dc voltage represents a nonlinear microwave impedance and may hence also be operated as a mixer. This was realized using the setup shown in Fig. 3. Two signals are fed to the junction simultaneously, one at frequency $f_{1}$ and the other at $f_{2}$. The signal at $f_{1}$ was derived from the $\operatorname{LO}\left(f_{1}=f_{\mathrm{LO}}\right.$ ) and $f_{2}$ (from a 12-18 GHz sweeper) was adjusted such that the difference frequency $f=f_{2}-f_{1}=f_{\mathrm{LO}} \pm f_{\mathrm{i} . \mathrm{f} \text {. }}$ or $f_{2}$ $=2 f_{\mathrm{LO}} \pm f_{\mathrm{1}_{\mathrm{f}} \mathrm{f}^{\circ}}$. The response at $f_{1}$ was of sufficient magnitude to be detected with a standard superheterodyne spectrometer (sensitivity $\sim 10^{-14} \mathrm{~W} / \mathrm{Hz}$ ). Microwave filters and isolators ensured that only pure monochromatic signals reached the junction and the balanced mixer. The output of the video detector was linear in incoming power at $f=f_{2}$ $-f_{1}$.

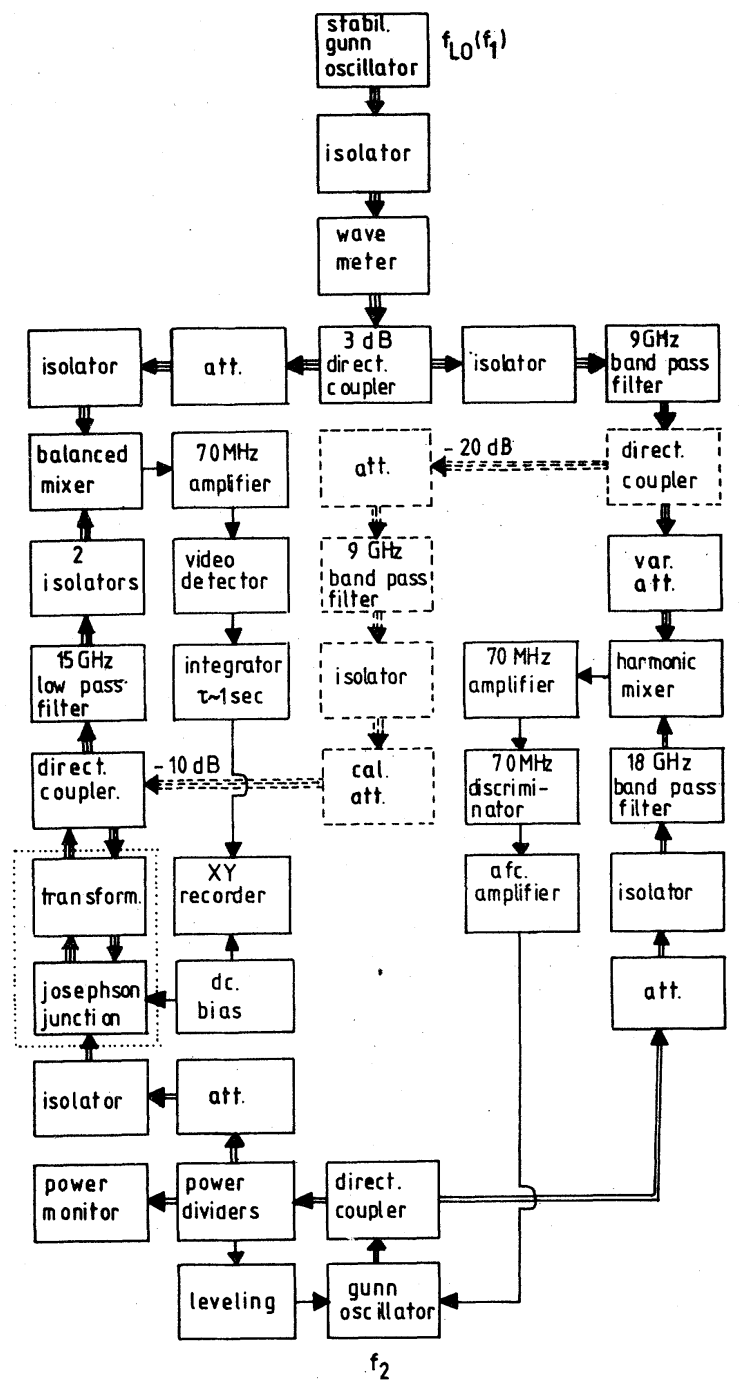

FIG. 3. Diagram of the microwave system for mixing $\left(f_{2}=2 f_{\text {LO }} \pm f_{\text {1.f. }_{0}}, f_{1}=f_{\mathrm{LO}}\right)$ and subharmonic generation. $\left[f_{2}=2\left(f_{\mathrm{LO}} \pm f_{i_{\text {.f. }}}\right)\right]$. Triple line: wave guide. Double line: microwave coaxial cable. Single line: low-frequency coaxial cable. The dashed path is used only in mixing experiments.

\section{Half-harmonic parametric oscillation}

The parametrically generated subharmonic os cillation has a fairly large amplitude ${ }^{13}$ (emitted power of order $10^{-12} \mathrm{~W}$ ) and may be detected using the spectrometer described above. Only one excitation frequency was used (the shunt drawn with dashed lines in Fig. 3 from the LO to the input coaxial line was disconnected) and the excitation frequency $f_{2}$ was readjusted such that $f_{2}=2\left(f_{\text {LO }}\right.$ $\left.\pm f_{\mathrm{i}_{\text {of }}}\right)$. The input power at $f_{2}$ must exceed a certain threshold value in order to generate the subharmonic oscillation. This threshold power can be calculated as a function of junction parameters. 
The threshold power plotted versus dc-bias current shall be referred to as the threshold curve.

\section{THEORY}

The experimental results are interpreted within the frame work of the simple shunted junction model, which states that the total current flowing in the junction may be expressed as a sum of four terms

$$
I_{\text {tot }}=I_{c} \sin \phi+\frac{V}{R}+\epsilon \frac{V}{R} \cos \phi+C \frac{d V}{d t} .
$$

Here, $I_{c}$ is the critical current, $\phi$ is the difference in phase between the macroscopic wave functions on either side of the barrier, $R$ is the shunt resistance, $C$ is the shunt capacitance, and $V$ is the voltage drop across the junction. The third term is the socalled quasiparticle-pair interference term or $\cos \phi$ term and is written as a phase dependent conductance with amplitude $\epsilon$ relative to the second term in (1). The phase difference $\phi$ and the voltage $V$ are related by

$$
V=\frac{\hbar}{2 e} \frac{d \phi}{d t},
$$

where $\hbar$ is Planck's constant $h / 2 \pi$ and $-e$ the electron charge.

We shall assume that the coefficients $I_{c}, R$, and $\epsilon$ are independent of the junction voltage. This is a reasonable approximation if only the junction voltage $|V| \ll \Delta / e$ and all frequencies involved $\ll 2 \Delta / h$. We shall further assume that the applied signals, dc and ac, are supplied by ideal current sources. Then, from Eqs. (1) and (2) the differential equation results

$\frac{\hbar C}{2 e} \frac{d^{2} \phi}{d t^{2}}+\frac{\hbar}{2 e R}(1+\epsilon \cos \phi) \frac{d \phi}{d t}+I_{c} \sin \phi=I_{0}+I_{\mathrm{ac}}(t)$.
In the present context we want to find solutions to Eq. (3) corresponding to zero dc voltage when the ac current has the form $I_{\mathrm{ac}}(t)=I_{1} \sin \left(\omega_{1}+\theta_{1}\right)+I_{2} \sin$ $\left(\omega_{2} t+\theta_{2}\right)$.

It is convenient to introduce the dimensionless quantities $j_{k}=I_{k} / I_{c}, \Omega_{k}=\omega_{k} / \omega_{0}, k=1,2, \ldots$, and $\tau$ $=\omega_{0} t$ where $\omega_{0} / 2 \pi$ is the maximum plasma frequency

$$
\omega_{0}=\left(2 e I_{c} / \hbar C\right)^{1 / 2} .
$$

Further, introducing the corresponding quality factor

$$
Q_{0}=\omega_{0} R C,
$$

we obtain the equation

$$
\begin{aligned}
& \ddot{\phi}+(1+\epsilon \cos \phi) \dot{\phi} / Q_{0}+\sin \phi \\
& =j_{0}+j_{1} \sin \left(\Omega_{1} \tau+\theta_{1}\right)+j_{2} \sin \left(\Omega_{2} \tau+\theta_{2}\right),
\end{aligned}
$$

where the dot means differentiation with respect to dimensionless time $\tau$.

$$
\text { A. Second-harmonic generation }\left(j_{1} \neq 0 ; j_{2}=0\right)
$$

With the monochromatic excitation at the frequency $\Omega_{1}$, we want to derive the voltage component at $2 \Omega_{1}$. This second-harmonic voltage may be found from Eq. (6) by inserting a trial solution of the form

$\phi(\tau)=\phi_{0}+\phi_{1} \sin \left(\Omega_{1} \tau+\psi_{1}\right)+\phi_{2} \sin \left(2 \Omega_{1} \tau+\psi_{2}\right)$.

The amplitude $\phi_{2}$ and the phase $\psi_{2}$ are determined self-consistently to second order in $\phi_{1}$ using the harmonic balance condition. Then the corresponding voltage components are determined by differentiation with respect to time [Eq. (2)].

The solution in terms of $\phi_{0}, \phi_{1}$, and $\phi_{2}$ is given by a set of coupled algebraic equations. Using complex notation

$$
\begin{aligned}
& j_{0}=J_{0}\left(\phi_{1}\right) \sin \phi_{0}, \\
& \phi_{1} e^{j \psi_{1}}=j_{1} e^{j \theta_{1}} /\left[\left(\frac{2 J_{1}\left(\phi_{1}\right)}{\phi_{1}} \cos \phi_{0}-\Omega_{1}^{2}\right)+j \frac{\Omega_{1}}{Q_{0}}\left(1+\frac{2 J_{1}\left(\phi_{1}\right)}{\phi_{1}} \epsilon \cos \phi_{0}\right)\right], \\
& \phi_{2} e^{j \psi_{2}}=\frac{\left(\frac{1}{2} \phi_{1} e^{j \psi_{1}}\right)^{2} \sin \phi_{0}\left(2 \epsilon \Omega_{1} / Q_{0}-j\right)}{\left[J_{0}\left(\phi_{1}\right) \cos \phi_{0}-4 \Omega_{1}^{2}\right]+j\left(2 \Omega_{1} / Q_{0}\right)\left[1+J_{0}\left(\phi_{1}\right) \epsilon \cos \phi_{0}\right]},
\end{aligned}
$$

where $J_{0}\left(\phi_{1}\right)$ and $J_{1}\left(\phi_{1}\right)$ are the zero- and firstorder Bessel functions of the first kind. To second order in $\phi_{1}$ we approximate $J_{0}\left(\phi_{1}\right) \simeq 1-\frac{1}{4} \phi_{1}^{2}$ and $2 J_{1}\left(\phi_{1}\right) / \phi_{1} \simeq 1-\frac{1}{8} \phi_{1}^{2}$. The complex second-harmonic voltage is simply $V_{2}=\left(\hbar \omega_{1} / e\right) \phi_{2} e^{j \psi_{2}}$, i.e., proportional to $\phi_{2} e^{j \psi_{2}}$. The factor $J_{0}\left(\phi_{1}\right)$ in Eq. (8a) expresses the microwave suppression of the critical current $\left[\sin \phi_{0}=1\right.$ for $\left.j_{0}=J_{0}\left(\phi_{1}\right)<1\right]$. It is seen from Eqs. (8a), (8b), and (8c) that the second-harmonic response may exhibit two resonance peaks as the dc-bias current is varied from zero to the critical value. Neglecting for a while $\frac{1}{4} \phi_{1}^{2}$ compared to one, we find one resonance at $\cos \phi_{0}=\Omega_{1}^{2}$ (provided $\Omega_{1}<1$ ) at a dc bias $j_{0}=\sin \phi_{0}=\left(1-\Omega_{1}^{4}\right)^{1 / 2}$ and another at $\cos \phi_{0}=4 \Omega_{1}^{2}$ (provided $\Omega_{1}<\frac{1}{2}$ ) at a lower value of (normalized) bias current $j_{0} \simeq(1$ 
$\left.-16 \Omega_{1}^{4}\right)^{1 / 2}$. We may introduce the junction plasma frequency which in normalized units becomes

$$
\Omega_{p}=\left(\cos \phi_{0}\right)^{1 / 2} \text {. }
$$

The resonances in the second harmonic response may then conveniently be referred to as the $\Omega_{p}=\Omega_{1}$ resonance and the $\Omega_{p}=2 \Omega_{1}$ resonance. The former may be observed if $\omega_{0}>\omega_{1}$ and the latter if $\omega_{0}$ $>2 \omega_{1}$.

$$
\text { B. Mixing }\left(j_{1} \neq 0 ; j_{2} \neq 0\right)
$$

With two signals at frequencies $\Omega_{1}$ and $\Omega_{2}$ applied simultaneously $\left(\Omega_{2}>\Omega_{1}\right)$ the nonlinear junction will generate sum and difference frequencies as well as higher harmonics. Assuming a solution to Eq. (6) of the form

$$
\begin{aligned}
\phi(\tau)=\phi_{0} & +\phi_{1} \sin \left(\Omega_{1} \tau+\psi_{1}\right)+\phi_{2} \sin \left(\Omega_{2} \tau+\psi_{2}\right) \\
& +\phi \sin (\Omega \tau+\psi)+\phi^{\prime} \sin \left(\Omega^{\prime} \tau+\psi^{\prime}\right),
\end{aligned}
$$

where $\Omega=\Omega_{2}-\Omega_{1}$ and $\Omega^{\prime}=\Omega_{2}+\Omega_{1}$, self-consistent expressions for the mixing products $\phi, \phi^{\prime}$ may be found to second order in $\phi_{1}, \phi_{2}$. Here, it is not necessary to include the higher harmonics of the applied frequencies since they will not contribute to $\phi, \phi^{\prime}$ to second order.

We shall not here present the full self-consistent second-order expressions for the frequency components included in Eq. (10). They are space consuming and not very instructive. Rather, we shall present a set of simplified expresssions which display the essential features of the solution

$$
\begin{aligned}
& j_{0}=\sin \phi_{0}=\left(1-\Omega_{p}^{4}\right)^{1 / 2}, \\
& \phi_{1}=j_{1}\left[\left(\Omega_{p}^{2}-\Omega_{1}^{2}\right)^{2}+\left(\frac{\Omega_{1}}{Q_{0}}\left(1+\epsilon \Omega_{p}^{2}\right)\right)^{2}\right]^{-1 / 2}, \\
& \phi_{2}=j_{2}\left[\left(\Omega_{p}^{2}-\Omega_{2}^{2}\right)^{2}+\left(\frac{\Omega_{2}}{Q_{0}}\left(1+\epsilon \Omega_{p}^{2}\right)\right)^{2}\right]^{-1 / 2},
\end{aligned}
$$

$$
\begin{aligned}
\phi= & \frac{1}{2} \phi_{1} \phi_{2} \sin \phi_{0} \\
& \times\left(\frac{1+\left(\epsilon \Omega / Q_{0}\right)^{2}}{\left(\Omega_{p}^{2}-\Omega^{2}\right)^{2}+\left[\left(\Omega / Q_{0}\right)\left(1+\epsilon \Omega_{p}^{2}\right)\right]^{2}}\right)^{1 / 2}, \\
\phi^{\prime}= & \frac{1}{2} \phi_{1} \phi_{2} \sin \phi_{0} \\
& \times\left(\frac{1+\left(\epsilon \Omega^{\prime} / Q_{0}\right)^{2}}{\left(\Omega_{p}^{2}-\Omega^{\prime 2}\right)^{2}+\left[\left(\Omega^{\prime} / Q_{0}\right)\left(1+\epsilon \Omega_{p}^{2}\right)\right]^{2}}\right)^{1 / 2},
\end{aligned}
$$

where we have introduced $\Omega_{p}^{2}=\cos \phi_{0}$. Equation (11a)-(11e) appear linearized and decoupled. In fact they are not due to additional factors $J_{0}(x)$, $2 J_{1}(x) / x$, with $x=\phi_{1}, \phi_{2}, \phi, \phi^{\prime}$ in front of all the $\sin \phi_{0}$ and $\cos \phi_{0}$ terms. The component at the difference frequency $\Omega=\Omega_{2}-\Omega_{1}$ [Eq. (11d)] exhibits resonances when $\Omega_{p}=\Omega, \Omega_{p}=\Omega_{1}$, and $\Omega_{p}=\Omega_{2}$ where $\Omega_{p}$ is determined by the dc-bias current $[\mathrm{Eq}$. (11a)].

$$
\text { C. Half-harmonic parametric oscillation }\left(j_{1}=0 ; j_{2} \neq 0\right. \text { ) }
$$

When the input power exceeds a certain threshold level subharmonic oscillations may be excited in the junction. The lowest threshold value occurs if the applied frequency $\Omega_{2}$ is near twice the plasma frequency and in that case the halfharmonic oscillation is generated. ${ }^{13}$ The threshold curve may be calculated as a function of junction parameters if a trial solution of the form

$$
\phi(\tau)=\phi_{0}+\phi_{2} \sin \left(\Omega_{2} \tau+\psi_{2}\right)+\delta \phi(\tau)
$$

is inserted into Eq. (6). To second order in $\phi_{2}$ an equation in $\delta \phi$ results which does not contain terms at dc or $\Omega_{2}$. The steady-state solution to the equation in $\delta \phi$ is the second harmonic at $2 \Omega_{2}$ (given by Eqs. (8a)-(8c) with index 1-2). The condition for the existence of a half-harmonic component at $\frac{1}{2} \Omega_{2}$ may be derived by requiring that the equation has a nonvanishing solution $\delta \phi_{2}$ $=\alpha \cos \frac{1}{2} \Omega_{2} \tau+\beta \sin \frac{1}{2} \Omega_{2} \tau$. Such a solution may exist if $\phi_{2} \geqslant \phi_{t}$, where

$$
\begin{aligned}
& \phi_{t}=2 \frac{\left(\left[J_{0}\left(\phi_{t}\right) \cos \phi_{0}-\left(\frac{1}{2} \Omega_{2}\right)^{2}\right]^{2}+\left\{\left(\Omega_{2} / 2 Q_{0}\right)\left[1+J_{0}\left(\phi_{t}\right) \in \cos \phi_{0}\right]\right\}^{2}\right)^{1 / 2}}{\left[2 J_{1}\left(\phi_{t}\right) / \phi_{t}\right] \sin \phi_{0}\left[1+\left(\epsilon \Omega_{2} / 2 Q_{0}\right)^{2}\right]^{1 / 2}}, \\
& j_{1}=\phi_{t}\left\{\left(\frac{2 J_{1}\left(\phi_{t}\right)}{\phi_{t}} \cos \phi_{0}-\Omega_{2}^{2}\right)^{2}+\left[\frac{\Omega_{2}}{Q_{0}}\left(1+\frac{2 J_{1}\left(\phi_{t}\right)}{\phi_{t}} \in \cos \phi_{\mathrm{b}}\right)\right]^{2}\right\}^{1 / 2}, \\
& j_{0}=J_{0}\left(\phi_{t}\right) \sin \phi_{0} .
\end{aligned}
$$

The minimum threshold is found at a dc bias where $\Omega_{p} \simeq \frac{1}{2} \Omega_{2}$ (recall that $\Omega_{p}^{2} \simeq \cos \phi_{0}$ ). In order to calculate the amplitude of the half-harmonic oscillation, terms of higher than second order in $\phi_{a}$ must be maintained in the expansion of the solution.

\section{EXPERIMENTAL RESULTS AND DISCUSSION}

We have measured the resonant microwave response of the junction biased at zero dc voltage by varying the dc-bias current from zero to the 
critical value.

Within the self-consistent second-order solutions presented above the critical current varies linearly with the applied microwave power $\left(J_{0}(\phi)\right.$ $\simeq 1-\frac{1}{4} \phi^{2}$, where $\phi^{2}$ is proportional to the input power). Accordingly, in the experiments the input power level was chosen so small that the critical current satisfied a linear power dependence.

Due to the nonlinear microwave response of the junction it is important to know the signal level inside the junction region in at least one bias point. At the critical current we must have $\sin \phi_{0}$ $=1$, which means that if the microwave signal at frequency, $\Omega_{k}$, is applied alone, the resulting phase modulation amplitude $\phi_{k}$, may be calculated at this bias point from $\phi_{k}=2\left(1-j_{c s}\right)^{1 / 2}$ when $j_{c s}$ is the (normalized) microwave suppressed critical current. The magnitude of the corresponding applied ac current $j_{k}$, may then be calculated from, e.g., Eq. (8b) assuming that the junction parameters $\omega_{0}$ and $Q_{0}$ are known. This calibration of the microwave input does not depend on the $\cos \phi$ amplitude $\epsilon$, since $\cos \phi_{0}=0$ at this particular bias point.

\section{A. Second-harmonic generation}

A typical recording of the complex second-harmonic voltage is shown in Fig. 4 . The excitation frequency was $f_{1}=4.403 \mathrm{GHz}$ and the figure shows 4 traces with a $90^{\circ}$ shift of the reference phase between the individual traces. Two orthogonal components (e.g., the $0^{\circ}$ and the $90^{\circ}$ traces) are sufficient to determine both the amplitude and the

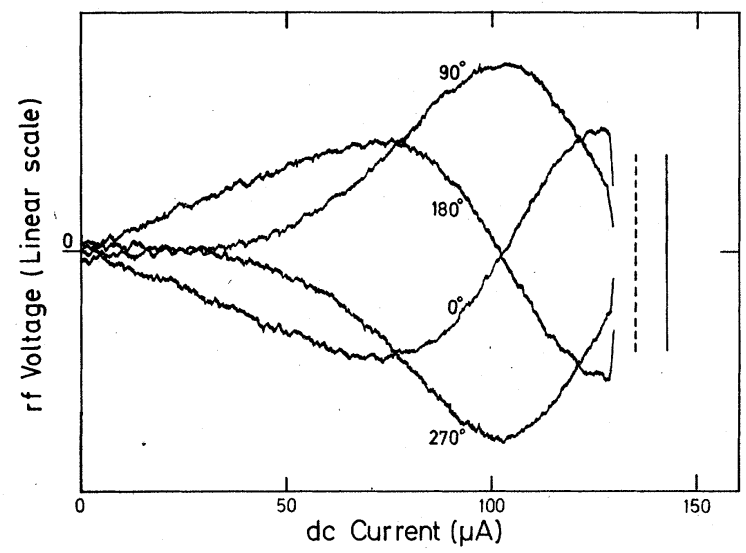

FIG. 4. Second-harmonic voltage as a function of the dc-bias current. Different phase shifter settings are indicated. The vertical lines show the critical currents with and without applied microwaves (dashed line and full line, respectively). $f_{1}=4.403 \mathrm{GHz} ; T / T_{\mathrm{c}}=0.990$; $T_{\mathrm{c}}=3.815 \pm 0.005 \mathrm{~K}$.

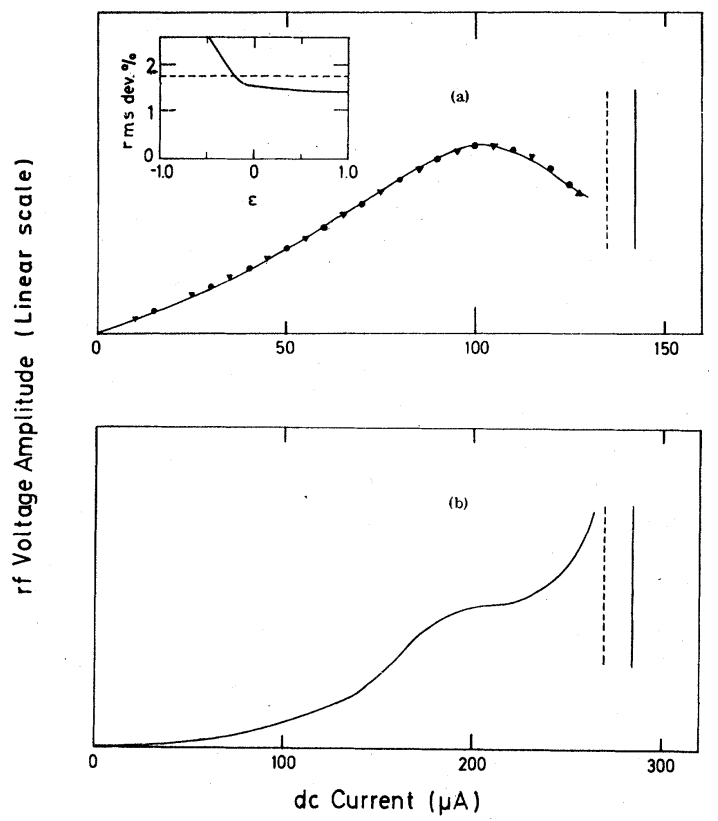

FIG. 5. (a) Amplitude curve determined from Fig. 4 (solid curve). The circles are calculated with $\omega_{0} / 2 \pi$ $=5.331 \mathrm{GHz}, Q_{0}=1.624$, and $\epsilon=0.2$. The triangles correspond to $\omega_{0} / 2 \pi=6.081 \mathrm{GHz}, Q_{0}=1.104$, and $\epsilon$ $=-0.2$. The inset shows the $\mathrm{rms}$ deviation minimized with respect to $\omega_{0}$ and $Q_{0}$ and the dashed horizontal line represents experimental uncertainty in percent of the peak value. (b) A typical amplitude curve showing the $\Omega_{p}=2 \Omega_{1}$ resonance at lower temperatures.

phase of the second-harmonic voltage. We did, however, always record the 4 traces since any deviation from a mirror-symmetric pattern due to drift of the junction temperature, the input power level, or the input frequency would be readily observed. Asymmetric patterns were rejected.

The amplitude curve ${ }^{14}$ derived from Fig. 4 is shown in Fig. 5(a). In this junction the condition $\left(\Omega_{1}<1\right)$ for observation of $\Omega_{1}=\Omega_{p}$ resonance was satisfied for temperatures $T / T_{c} \lesssim 0.994\left[\Omega_{1}=\right.$ $\omega_{1} / \omega_{0}$, where $\omega_{0}$ is given by Eq. (4); the critical current and hence $\omega_{0}$ increases as the temperature decreases]. As the temperature was decreased further the dc-bias current corresponding to the $\Omega_{p}=\Omega_{1}$ resonance peak approached the critical current and for temperatures $T / T_{c} \lesssim 0.982$ the junction would switch to the finite voltage state before the resonance was traced out. Hence, useful recordings of the $\Omega_{p}=\Omega_{1}$ resonance was obtained only in the temperature interval $0.994 \geqslant T / T_{c} \geqslant 0.982$.

In order to determine the junction parameters $\omega_{0}, Q_{0}$, and $\epsilon$ we have evaluated the expressions (8a) $-(8 c)$ on a digital computer and calculated the amplitude of the second-harmonic voltage as a function of bias current. For a series of $\epsilon$ values 
in the interval $|\epsilon|<1$ we have made a least mean square fit to the experimental curve by variation of $\omega_{0}$ and $Q_{0}$. Thus, the values which minimize the rms deviation, i.e., reproduces the peak position and the peak width depend on $\epsilon: \omega_{0}=\omega_{0}(\epsilon)$, $Q_{0}=Q_{0}(\epsilon)$. As demonstrated in Fig. 5(a) an excellent fit is possible for a several $\epsilon$ values. The circles in Fig. 5(a) are calculated with $\epsilon=0.2$, $\omega_{0} / 2 \pi=5.331 \mathrm{GHz}$, and $Q_{0}=1.624$ and the triangles with $\epsilon=-0.2, \omega_{0} / 2 \pi=6.081 \mathrm{GHz}$, and $Q_{0}=1.104$. The corresponding rms deviations are 1.55 and 1.80 , respectively, in percent of the peak amplitude. For comparison the experimental uncertainty allows an rms deviation $\$ 1.84$. The inset in Fig. 5(a) shows the rms deviation versus $\epsilon$ using the optimum values of $\omega_{0}(\epsilon)$ and $Q_{0}(\epsilon)$. Obviously, additional information is needed in order to determine all three parameters.

At lower junction temperatures the condition $\left(\Omega_{1}<\frac{1}{2}\right)$ for observation of the $\Omega_{p}=2 \Omega_{1}$ resonance was satisfied [according to Eq. (4) this requires an increase of the critical current by a factor of 4]. Due to the low $Q$ of the plasma resonance the $\Omega_{p}=2 \Omega_{1}$ resonance appeared as a badly defined shoulder on the low current side of the $\Omega_{p}=\Omega_{1}$ resonance peak as shown in Fig. 5(b) obtained on another junction. Such recordings were not useful.

\section{B. Mixing}

A typical recording of the generated power at $f=f_{2}-f_{1}=8.599 \mathrm{GHz}$ is shown in Fig. 6. The excitation frequencies were $f_{2}=17.268 \mathrm{GHz}$ and $f_{1}=$ $f_{\mathrm{LO}}=8.669 \mathrm{GHz}$. With this detection scheme use-

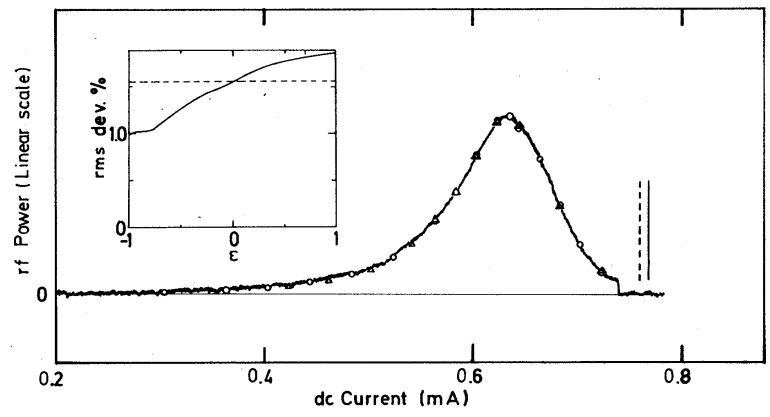

FIG. 6. Lower sideband mixing component as a function of the dc-bias current. The vertical lines show the critical currents with no applied microwave signal and with the two signals applied simultaneously (full and dashed lines, respectively). $f_{2}=17.268 \mathrm{GHz}, f_{1}=8.669$ $\mathrm{GHz}$, and $T / T_{c}=0.942$. The circles are calculated with $\omega_{0} / 2 \pi=13.104 \mathrm{GHz}, Q_{0}=1.832$, and $\epsilon=-0.8$. The triangles: $\omega_{0} / 2 \pi=12.091 \mathrm{GHz}, Q_{0}=3.498$, and $\epsilon=0$. The inset shows the rms deviation minimized with respect to $\omega_{0}$ and $Q_{0}$ and the dashed horizontal line represents experimental uncertainty in percent of the peak value. ful resonances were observed in the temperature interval $0.965 \geqslant T / T_{c} \geqslant 0.930$ where the plasma frequency $\Omega_{p} \simeq \Omega, \Omega_{1}$. At the same time, however, $\Omega_{p} \sim \frac{1}{2} \Omega_{2}$ which means that the threshold for the onset of parametric oscillations at $\frac{1}{2} \Omega_{2}$ may be reached even for values of input power at $\Omega_{2}$ where the second-order theory is still valid. Furthermore, at power levels just below the threshold, part of the detected signal may consist of parametrically amplified noise (cf. Sec. IV C). Since these effects are not accounted for in the theoretical expresssion Eqs. (11a)-(11e) we applied only signal levels far below the threshold.

The junction parameters were determined by performing a least mean square fit to the experimental results as described in Sec. IV A. The calibration of the microwave input was made by measuring the microwave suppressions of the critical current with the signals at $\Omega_{1}$ and $\Omega_{2}$ applied separately. The parameters which minimize the rms deviation from the trace in Fig. 6 are $\epsilon=0.8$, $\omega_{0} / 2 \pi=13.104 \mathrm{GHz}$, and $Q_{0}=1.832$. The calculated response with these parameters is shown as circles in Fig. 6. The rms deviation is $\mathbf{1 . 0 2}$ in percent of the peak power value and the experi-

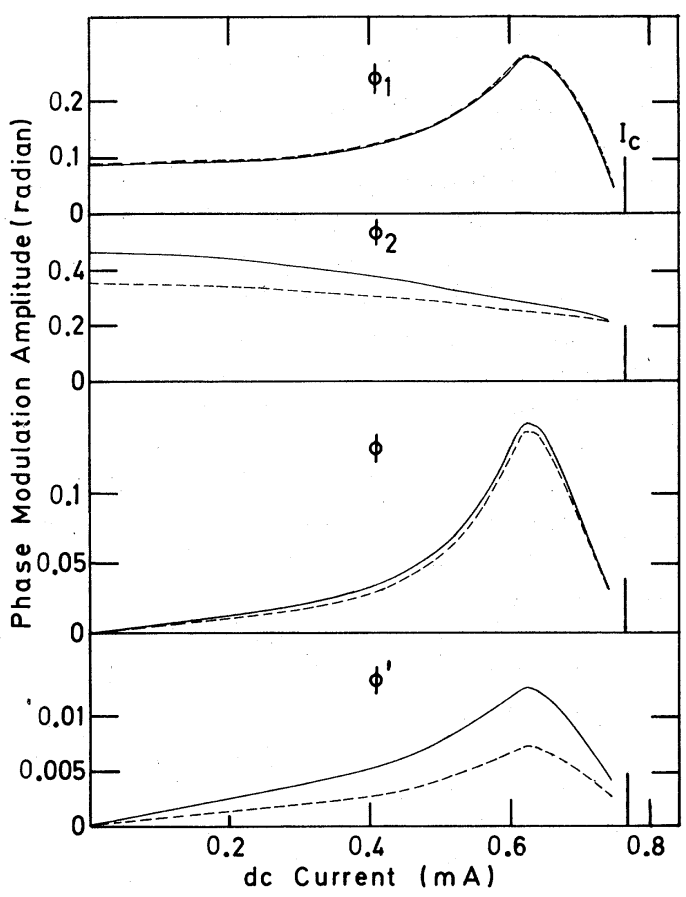

FIG. 7. Phase modulation amplitudes $\phi_{1}, \phi_{2}, \phi$, and $\phi^{\prime}$ at frequencies $f_{1}, f_{2}, f_{2}-f_{1}$, and $f_{2}+f_{1}$ corresponding to the fit on Fig. 6 . Full curves: $\omega_{0} / 2 \pi$ $=13.104 \mathrm{GHz}, Q_{0}=1.832$, and $\epsilon=-0.8$. Dashed curves: $\omega_{0} / 2 \pi=12.091 \mathrm{GHz}, Q_{0}=3.498$, and $\epsilon=0$. Critical current without applied signals is indicated. 
mental uncertainty allows 1.55 in the same units. The triangles correspond to $\epsilon=0, \omega_{0} / 2 \pi=12.09$ $\mathrm{GHz}$, and $Q_{0}=3.498$. As illustrated in the inset also here the rms deviation is below the experimental uncertainty for a range of $\epsilon$ values $(-1 \lesssim \epsilon$ $\lesssim 0)$.

We have calculated the phase modulation amplitudes $\phi, \phi^{\prime}, \phi_{1}$, and $\phi_{2}$ corresponding to the experimental data in Fig. 6. The results with the two different sets of parameters $\left(\epsilon, \omega_{0}, Q_{0}\right)$ discussed above are shown in Fig. 7(a)-7(d). From Fig. 7 we find that the phase modulation amplitudes of the frequency components all satisfy $\frac{1}{4} \phi_{i}^{2} \ll 1$ such that the self-consistent second-order solution to Eq. (6) is expected to hold.

According to Eqs. (11c) and (11d) the mixing component at $\Omega$ should exhibit a resonance also at $\Omega_{p}=\Omega_{2}$, i.e., at temperatures where the plasma frequency is $17-18 \mathrm{GHz}$. Unfortunately, this resonance could not be resolved since the response still was dominated by the $\Omega_{p}=\Omega, \Omega_{1}$ resonance. At these low temperatures the latter resonance could not be recorded because the peak occurred too close to the critical current. [The traces typically appeared somewhat similar to Fig. (5b).]

\section{Half-harmonic parametric oscillations}

A sequence of recordings of the parametrically generated oscillations at $\frac{1}{2} f_{2}=8.708 \mathrm{GHz}$ is shown in Fig. 8 stacked on the bias current versus pump power plane. Similar curves showing the existence of a threshold power for the onset of subharmonic oscillations have been reported previously. ${ }^{13}$ One difference between the present data and those of Ref. 13 is the absence of hysteresis; this is due to the relatively large losses in the present junctions. The intention was to use the shape of the experimental threshold curve to obtain information on $\epsilon$ by comparison with the theoretical result [cf. Eqs. (13a)-(13c)]. Unfortunately, no straight forward interpretation of these experimental traces is possible. The complications arises due to a phenomenon illustrated in Figs. 9(a)-9(c), which shows a spectrum analyzer display of the frequency distribution of the i.f. amplifier output. The three curves correspond to a fixed input power level and different biasicurrents defined in the inset which shows a typical recording from Fig. 8.

For a bias current well outside the region of subharmonic oscillations (the arrow a in the insert) the corresponding i.f. amplifier spectrum is shown in Fig. 9(a). For a bias current close to the peak of subharmonic oscillations (the arrow $c$ in the insert) the subharmonic oscillation is seen as a narrow line with a frequency width compar-

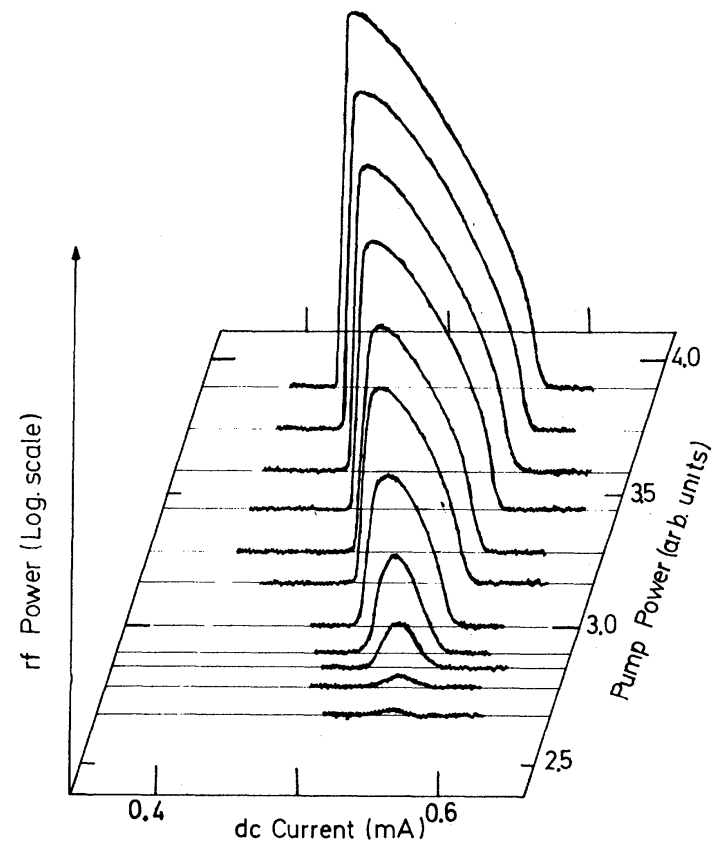

FIG. 8. Experimental parametric half-harmonic power shown in the bias current versus pump power plane. The ordinate is the power received at the frequency $\frac{1}{2} f_{2} ; f_{2}=17.416 \mathrm{GHz}, I_{c}=0.742 \mathrm{~mA}$, and $T / T_{c}$ $=0.942$.

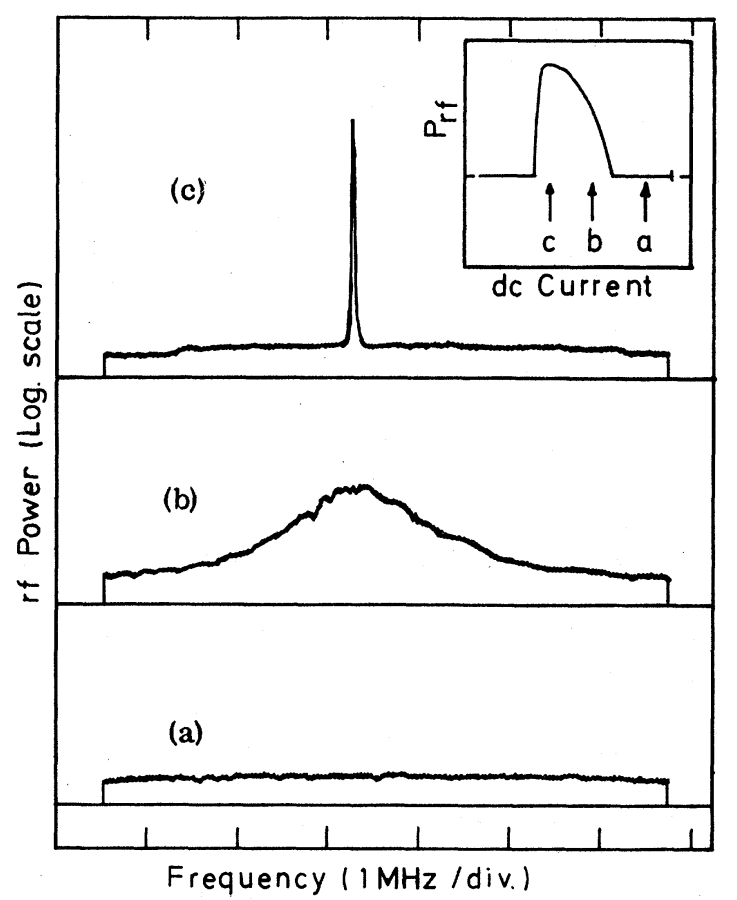

FIG. 9. Typical spectrum analyzer displays of the frequency distribution in the i.f. amplifier output corresponding to the curves in Fig. 8. The bias currents corresponding to $\mathrm{a}, \mathrm{b}$, and $\mathrm{c}$ are indicated by the arrows in the inset. 
able to the width of the pump source. However, in an intermediate situation (e.g., at the arrow b in the inset) the corresponding i.f. amplifier output Fig. 9(b) shows a rise in the background noise forming a very wide peak. This spectrum shows parametrically amplified noise. ${ }^{15}$ It should be mentioned that with the same junction we have measured a parametric gain at the top flange of $16 \mathrm{db}$ at $X$-band frequencies. ${ }^{15}$ The results of the noise amplification effect is that the signal detected in the $10 \mathrm{MHz}$ bandwidth of the i.f. amplifier varies fairly smoothly with dc current without any distinct features displaying the crossing of the threshold curve given by the "noise free" theory.

Nevertheless, we made an analysis from the series of curves in Fig. 8 using arbitrarily for the threshold curve the values of the bias current where the detected signal rises above the base line. Fitting this experimental threshold curve to the set of equations [Eqs. (13a)-(13c)] by means of a computer in the same way as in Secs. IV AIV $B$ we were able to obtain a satisfactory agreement between theory and experiment for almost any value of $\epsilon$ between -1 and +1 . The sets of parameters $\omega_{0}, Q_{0}$, and $\epsilon$ obtained this way were consistent with the values obtained by mixing (Sec. IV B) in the same temperature range. However, because of the uncertainty due to parametric noise amplification these data are not used in the final evaluation of $\epsilon$.

\section{Determination of the $\cos \phi$ amplitude}

As already mentioned additional information is necessary in order to determine the junction parameters. The temperature dependence of either $\omega_{0}$ or $Q_{0}$ may provide such information. According to Eqs. (4) and (5) both quantities vary as $I_{c}^{1 / 2}$. The junction capacity may reasonably be assumed independent of temperature in the narrow temperature interval of the present experiment, since $C$ is determined only by barrier properties such as dielectric constant and barrier thickness. Hence, $\omega_{0} \propto\left[I_{c}(T)\right]^{1 / 2}$. The temperature dependence of the quality factor $Q_{0}$ is more difficult to predict because the effective loss resistance may depend on temperature (and frequency ${ }^{16}$ ). For this reason only the theoretical temperature dependence of $\omega_{0}$ shall be used. In Fig. 10 the values of $\omega_{0}$ determined from the fitting procedure is plotted versus $I_{c}^{1 / 2}$ with $\epsilon$ as a parameter. The points (a) and (b) in the figure and the condition that $|\epsilon|<1$ everywhere define the two straight lines drawn from the origin and determine the lower and upper limit of the capacity, $334 \leqslant C \leqslant 370 \mathrm{pF}$. From Eq. (4) we calculate the corresponding upper and lower limit of $\omega_{0}$ for each value of $I_{c}$. Finally, the range of

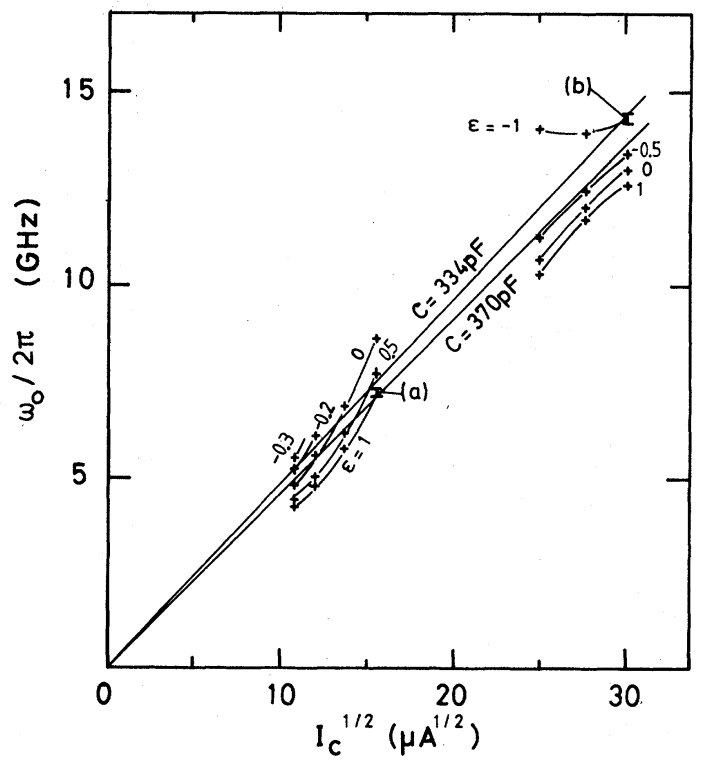

FIG. 10. Maximum plasma frequency, $\omega_{0} / 2 \pi$ vs square root of the critical current with $\epsilon$ as a parameter. The slope of the two straight lines drawn from the origin determines the extremal values of the junction capacity: $334 \leqslant C \leqslant 370 \mathrm{pF}$.

possible $\epsilon$-values is determined from the dependence $\omega_{0}=\omega_{0}(\epsilon)$ which was established above and illustrated in Figs. 11(a) and 11(b) valid for the crucial values of $I_{c}^{1 / 2}=15.6 \mu \mathrm{A}^{1 / 2}$ and $I_{c}^{1 / 2}=30.1$ $\mu \mathrm{A}^{1 / 2}$. In the lower part of Fig. 11 the corresponding rms deviations are shown versus $\epsilon$. We find that the variation of the rms deviations are consistent with the values of $\epsilon$ determined from the $\omega_{0}(\epsilon)$ dependence in the upper part of Fig. 11. This agreement was found to hold for all the experimental traces. In Fig. 12 the resulting amplitude, $\epsilon$ of the $\cos \phi$ conductance is shown versus critical current and reduced temperature (lower and upper horizontal scales, respectively). The experimental uncertainty indicated by the error bars is considerable but nevertheless the figure shows unambiguously that at the highest temperatures $\epsilon$ is positive and increasing with decreasing temperature. At still lower temperatures the sign has changed and $\epsilon$ approaches -1 at the lowest temperatures of the present experiment.

We are not able to give a strict theoretical explanation for this change in $\epsilon$ with temperature. Qualitatively, we suggest that the observed dependence may be the result of at least two competing mechanisms. The "quasiparticle-pair" interference current taken into account by the tunneling calculation in the adiabatic approximation is responsible for the positive sign at high temperatures. At low temperatures some kind of relaxation process may be responsible for the negative 

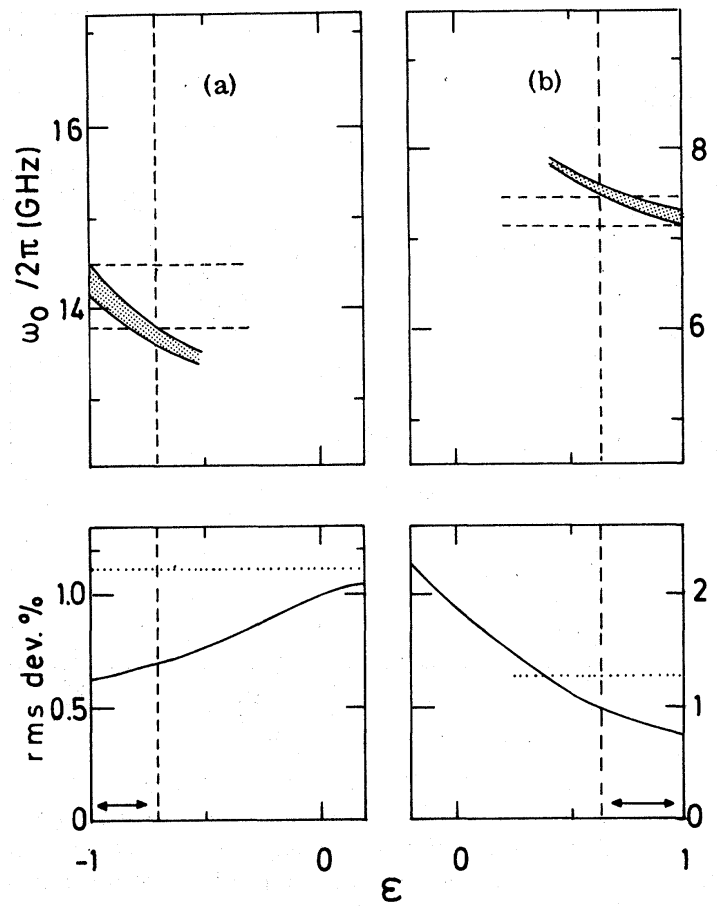

FIG. 11. Determination of $\epsilon$ for the two crucial values of $I_{c}$ in Fig. 10 indicated with (a): $I_{c}^{1 / 2}=30.1 \mu \mathrm{A}^{1 / 2}$ (mixing), (b): $I_{c}^{1 / 2}=15.6 \mu A^{1 / 2}$ (second harmonic). The upper part of the figures show the function $\omega_{0}(\epsilon)$ intersecting the two dashed lines determined by a calculation of $\omega_{0}$ with $C=334$ and $370 \mathrm{pF}$. [The function $\omega_{0}(\epsilon)$ is represented by the shaded band reflecting the experimental accuracy]. The lower part of (a) and (b) shows the rms deviations (minimized with respect to $\omega_{0}$ and $Q_{0}$ ). The dotted horizontal line represents the experimental uncertainty, and the horizontal arrow shows the range of $\epsilon$ values consistent with the capacity values and the condition $|\epsilon| \leqslant 1$.

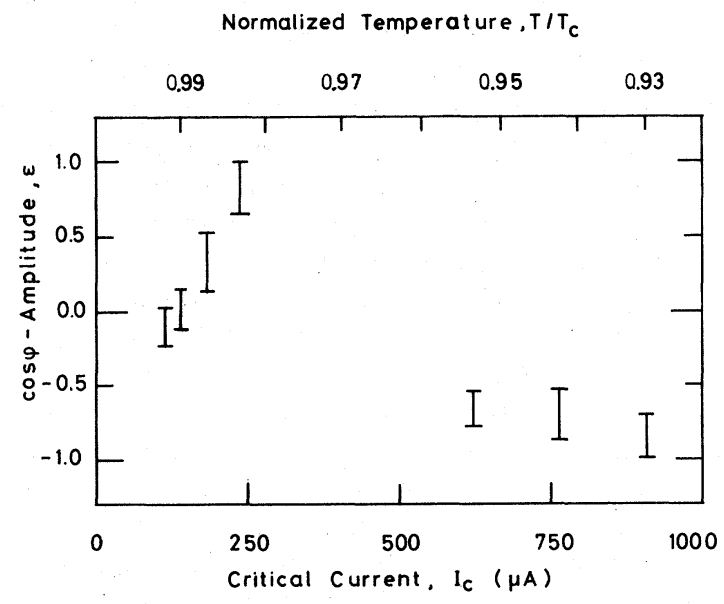

FIG. 12. Measured $\cos \phi$ amplitude vs junction critical current and normalized temperature.

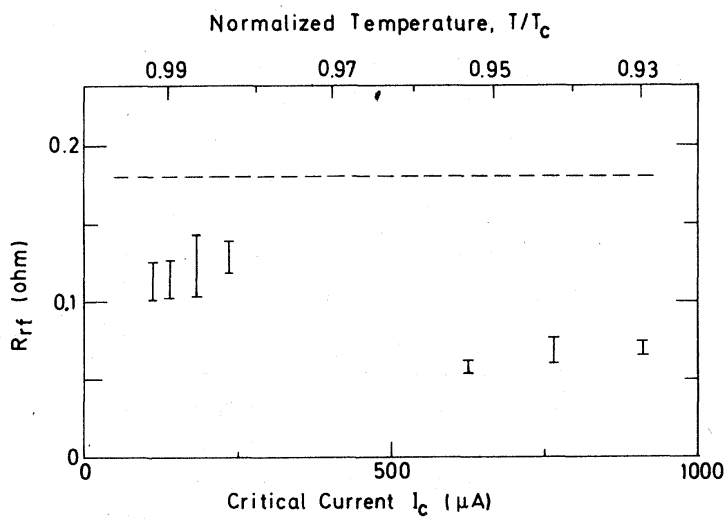

FIG. 13. Measured $\mathrm{rf}$ resistance $R_{\mathrm{rf}}$ as a function of junction critical current and normalized temperature. The dashed line is the normal-state dc resistance $R$ $=0.18 \Omega$.

sign. As is well known ${ }^{9,10}$ the introduction of a (pair) relaxation time $\tau$ gives rise to two extra current channels. One is of Ohmic character and the other has a structure similar to the $\cos \phi$ current, with a negative sign, 9,10 however. Both of these contributions are proportional to $I_{c} \tau \cdot{ }^{9,10}$ That this is the case may be illustrated by a simple argument by Stephen ${ }^{17}$; he writes for the pair current:

$I_{p}(t)=I_{0} \sin \phi(t-\tau) \simeq I_{0} \sin \phi(t)-(2 e / \hbar) I_{0} \tau \cos \phi V$.

Assuming that $\tau$ is proportional to $\Delta^{-1}$ and $I_{0}$ is proportional to $\Delta^{2}$ close to $T_{c}$ such a relaxation contribution would increase as $\Delta(T)$ giving zero contribution close to $T_{c}$ and becoming of increasing importance as the temperature is lowered. An explanation along these lines is further supported by Fig. 13 which shows the $r f$ resistance of the junction as obtained from the fitted values of $Q_{0}$. Although the uncertainty is large, the data may be interpreted in terms of the opening of an extra Ohmic current channel giving rise to a reduced shunt resistance at low temperatures. If such an intuitive and qualitative explanation is correct it will imply that the adiabatic approximation used in the tunneling Hamiltonian calculation is not justified in relation to the present experiments.

\section{SUMMARY AND CONCLUSION}

We have studied the temperature dependence of the resonant microwave response at the plasma frequency of tunnel junctions current biased at zero dc voltage. The plasma oscillations were excited using three different schemes all of which produced a resonant response at the receiver frequency ( $9 \mathrm{GHz})$ : (a) second-harmonic generation 
with excitation at $\sim 4.5 \mathrm{GHz}$, (b) mixing with input signals at $\sim 9$ and $\sim 18 \mathrm{GHz}$, and (c) half-harmonic parametric oscillations excited by a $\sim 18 \mathrm{GHz}$ signal. In this way measurements on each individual junction were possible in two temperature intervals $0.994 \geqslant T / T_{c} \geqslant 0.982$ and $0.965 \geqslant T / T_{c} \geqslant 0.930$ corresponding to plasma frequencies $\sim 4.5$ and $\sim 9$ $\mathrm{GHz}$, respectively.

By comparing the experimental results with calculations based on the shunted junction model we found that the phase dependent conductance or $\cos \phi$ conductance of superconducting tunnel junctions was positive at temperatures just below $T_{c}$ and that the relative amplitude increased from near zero towards +1 as the temperature was decreased. This is in qualitative agreement with the tunneling Hamiltonian calculation. At lower temperatures, however, the sign had changed and the amplitude approached -1 .

In order to understand this behavior we conclude that the observed temperature dependence of the $\cos \phi$ amplitude may be the result of a balance between at least two different mechanisms. The "quasiparticle-pair" interference taken into account by the tunneling calculation is responsible for the positive sign and at lower temperatures other mechanisms, e.g., some kind of relaxation process, dominate the behavior and explain the negative sign.

\section{ACKNOWLEDGMENTS}

We want to express our gratitude to M. Pallisgaard of the Electromagnetic Institute, T. Guldbrandsen of Physics Laboratory III both at The Technical University, and M. T. Levinsen of Physics Laboratory I. We want to express our gratitude to the $\mathrm{H}$. C. Oersted Institute for their willingness to lend us microwave components. We are further grateful to T. F. Finnegan for his comments and suggestions at the initial stages of this work.
${ }^{1}$ N. F. Pedersen, T. F. Finnegan, and D. N. Langenberg, Phys. Rev. B $\underline{6}, 4151$ (1972).

${ }^{2}$ B. D. Josephson, Adv̄. Phys. 14, 419 (1965).

${ }^{3}$ U. K. Poulsen, Rev. Phys. Appl. 9, 41 (1974).

${ }^{4}$ R. E. Harris, Phys. Rev. B 10,84 (1974).

${ }^{5}$ D. A. Vincent, B. S. Deaver, Jr., Phys. Rev. Lett. 32 , 212 (1974); R. Rifkin, D. A. Vincent, B. S. Deaver, Jr., and P. K. Hansma, J. Appl. Phys. 47, 2645 (1976).

${ }^{6}$ C. M. Falco, W. H. Parker, and S. E. Trullinger, Phys. Rev. Lett. 31, 933 (1973); 31, 1476 (1973); M. Nisenoff and S. Wolf, Phys. Rev. B 12, 1712 (1975).

${ }^{7} \mathrm{M}$. R. Halse and J. C. Taunton, in SQUID, edited by H. D. Hahlbohm and H. Lübbig (de Gruyter, Berlin and New York, 1977), p. 71.

${ }^{8}$ O. P. Balkashin and I. Y. Yanson, Fiz. Nizk. Temp. 2, 289 (1976).

${ }^{9}$ B. S Deaver, Jr., R. G. Boone, and R. Rifkin, Phys. Lett. A 57, 186 (1976).

${ }^{10}$ H. Højgaard Jensen and P. E. Lindelof, J. Low Temp. Phys. 23, 469 (1976).

${ }^{11}$ Kazuo Hido and Yoshiyuki Ono, J. Low Temp. Phys. 29, 355 (1977).
${ }^{12}$ D. D. Coon and M. D. Fiske, Phys. Rev. 138, A744 (1965); J. T. Chen, T. F. Finnegan, and $\overline{D . ~ N . ~ L a n g e n-~}$ berg, Physica (Utr.) 55, 413 (1971).

${ }^{13} \mathrm{~J}$. Mygind, N. F. Pedersen, and O. H. Soerensen, Appl. Phys. Lett. 29, 317 (1976).

${ }^{14}$ The phase information also contained in the experimental traces has not been used. It turned out, however, that no additional information may be derived from the measured phase shifts.

${ }^{15} \mathrm{~J}$. Mygind, N. F. Pedersen, and O. H. Soerensen, Appl. Phys. Lett. B 1, 17 (1978).

${ }^{16}$ The effective loss resistance may depend also on the applied microwave power level and may include losses such as the temperature- and frequency-dependent surface losses in the superconducting films. Hence, the resistance $R$ in the quasiparticle term $V / R$, and in the $\cos \phi$ term, $\epsilon V \cos \phi / R$, may actually be different. This means that the magnitude of $\epsilon$ which is determined here may not be quite correct. The sign of $\epsilon$ is, however, not affected.

${ }^{17}$ M. J. Stephen, Phys. Lett. A $\underline{46}, 289$ (1973). 